\title{
Development Correlations of the Buds of Grapevine (Vitis vinifera L.)
}

\author{
Liliana ROTARU \\ University of Agricultural Sciences and Veterinary Medicine, Faculty of Horticulture, 3 Mihail Sadoveanu Al., Iasi 700490, \\ Romania; lirotaru@uaiasi.ro
}

\begin{abstract}
The development characteristics of different buds of the grapevine are mainly related by stimulation and/or inhibition effects, the action of which is still inexplicable. The present study examines the development dynamics of the buds of a one-year old branch after excision of different buds and the application of $\alpha$-naphtyl acetic acid (ANA), as well as the growth capacity of each bud individually. We verified the effects of acrotony cited previously by various researchers. These effects are due to different developmental characteristics of which could to lay the groundwork for the improvement of different productions methods.
\end{abstract}

Keywords: buds, excision, grapevine, Muscat Ottonel, $\alpha$-naphtyl acetic acid

\section{Introduction}

The correlations of growth are related with stimulation and/or inhibition which exist between the various buds of the vegetative apparatus.

In previous works on the correlations on the one-year old branch of the woody plants, Bessis (1965), Champagnol (1984) and Vincent (1995) estimated that type of ramification of the considered species was important, which was determined by interaction of at least three factors:

- fixed properties; installation during the preceding vegetative cycle and which make that the development of a bud seems more or less given by its situation on the branch according to a gradient basitone or acrotone, the degree of intensities this fixing being in addition variable with the species;

- inhibitions related to "current" nature; which can either depend on trophic and/or hormonal situation, and of which result in competition between buds. These inhibitions are revealed by the size of the fruit trees, and the "current" refers to the substances or influences that exist in the stem at the time when inhibition is noted;

-the (physical) strength; of which the ascending values lead, for many trees, with the development of a greater number of buds, and who can thus thwart the two precedent factors.

Huglin (1998) denotes that contribution of these factors for vine is mainly due to the fixed properties. On the other hand Reynier (2000) emphasizes inhibitions of "current" nature with growth differences of buds from different rows on the same vine shoot after an unequal disbudding.

The tendency of acrotony is known for a long time. A main feature at the branch scale is acrotony, usually defined as the increase in vigor (length, diameter, number of leaves) of the vegetative proleptic branches (from dormant buds) from the bottom to the top position of the parent growth unit (Bell, 1991; Lauri and Lespinasse, 2001; Brunel et al., 2002). Acrotony typically gives rise to a whorl of branches, defining the rhythmicity of branching, which is a major determinant of plant architecture (Lauri, 2007).

Adrian et al. (1996), Bessis and Fournioux (1992) and Fournioux (2001) mentioned that, in the case of short branches with fruits (in 2 eyes for example), the higher eye which is the first one in vegetation results in a branch significantly vigor than the lower branch. For longer branches with fruits, not arched, the order of development is the same (Goffinet, 1991; Fournioux, 1998; Williams, 2000; Vasconcelos et al., 2009).

The objective of the experiment is the decribe the main features of growth correlations between the latent buds of a one-year old vine shoot besides with the effect of using ANA on growth parameters.

\section{Material and methods}

Three hundred rods of Muscat Ottonel grapevine variety were taken during the winter, in an experimentation parcel, University of Agricultural Sciences and Veterinary Medicine, Iasi. Two experimental series were installed. The 1 st series included two batches. For the 1st batch, 30 rods of 8 buds were used and the base wood section was 2-year old shoot. These rods were cutted in pots, with the 2-year old parts planted in vermiculite and maintained vertical by the help of a "perch" fastener. The 2 nd batch consists of 30 rods on which had an eye for each row. The cuttings were in parallel lines following the order of insertion of the eyes, in vermiculite pots. The 2 nd series was made up of eight batches. Each batch includes 30 cuttings with 4 eyes, 

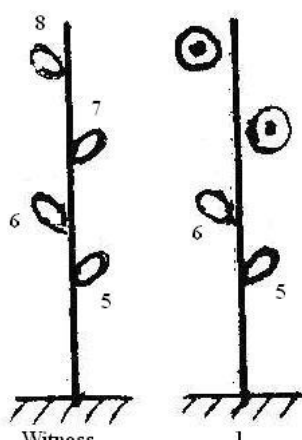

1

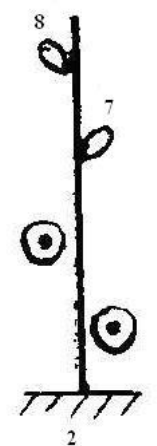

2

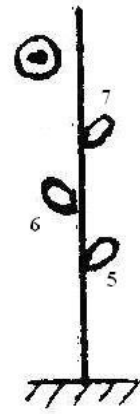

3

node sightless

- ANA $1 \mathrm{mg} \mathrm{L}^{-1}$

Fig. 1. Experimental model
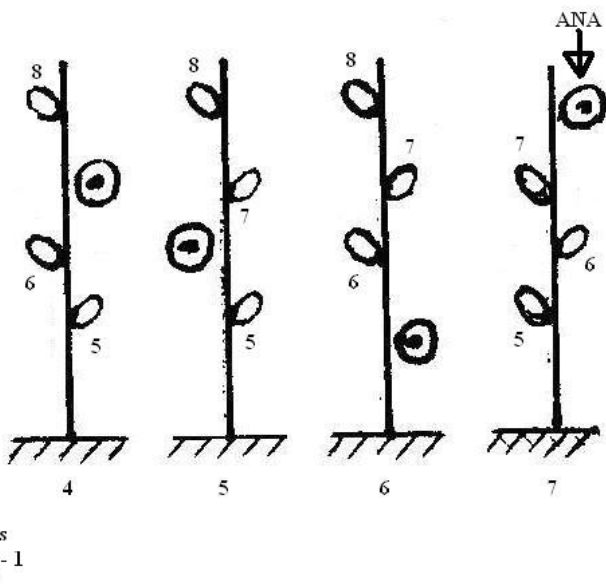

of 5-6-7-8 rows, placed in culture out of pot on vermiculite ( 3 cuttings per pot). In each batch, the variable was the number of eyes (Fig.1).

All the cuttings were exposed to same culture conditions: the temperature was $25^{\circ} \mathrm{C}$ for the day time and $22^{\circ} \mathrm{C}$ for the night; illumination was supplied with sodium lamps resulting in 16 hours photoperiod for the day time and 8 hours harms; irrigation was done regularly.

The cuttings were put in culture on $16^{\text {th }}$ December. The first statement was carried out as soon as the buds started to strip and finished with the fall as of the apex. Seven readings were taken. The application of ANA, $\alpha$-naphtyl acetic acid, 10-3 g/l was repeated once a week.

\section{Results and discussion}

Series I-Batch 1: rods in 8 eyes (Fig. 2)

The heterogeneity in the lengths of growth of the branches, according to the row of bud insertion, is significant. There are 3 growth groups: (i) hardly developed (buds 1 and 2), (ii) weak growth (buds 3 to 6), and (iii) high growth (buds 7 and 8 ). The growth in buds 3 to 6 was weak but equal and the growth which had a very considerable growth and in particular that of row 8 .

According to Huglin (1998) the tendency to acrotone in plant, meaning the strength, is low when winter conditions are rigorous. The length of branches mainly depends on natural or artificial hydrous mode. In addition, the light is effective quantitatively through photosynthesis and qualitatively by photoperiods (Bessis, 1986).

In table 1 growth averages of shoots are given. It is observed that the speed of budding is faster for the top, average for median and slow for base buds.

This phenomenon is the acrotony of budding, studied by Bessis (1986) which might be the result of variability in dormancy parameters. According to Huglin (1998) and Galet (1993) the speed of disbudding varies according to air temperature, whereas Huglin (1998), mentions the importance of vine variety.
The differences between the various growths increase with the wire of time, in absolute value. Here, it appears that in relative value these variations remain equivalent. What translates by the fact that if a growth of doubled row 1 length, a growth of row 8 too.

\section{Batch 2: Buds cut up in cutting of an eye}

It is noted that on 240 buds, 228 stripped; representing $95 \%$ of budding (Fig. 3), meaning that a separate bud from any row has the potential of an identical budding.

Series II-Correlations of growth between 4 successive buds

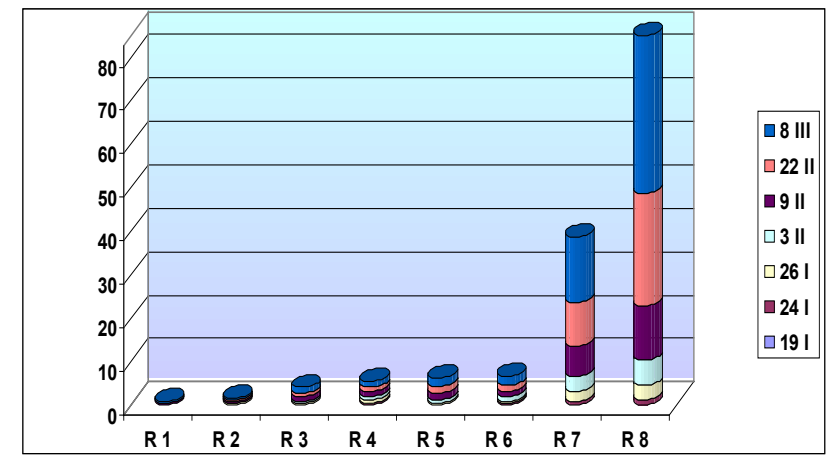

Fig. 2. Final average length of the shoots, in $\mathrm{cm}$, resulting from the various buds according to their row of insertion

Tab. 1. Growth stocks average of the shoots, in $\mathrm{cm}$

\begin{tabular}{cccccccc}
\hline \multirow{2}{*}{$\begin{array}{c}\text { Number } \\
\text { of rope }\end{array}$} & $14 / \mathrm{I}$ & $19 / \mathrm{I}$ & $26 / \mathrm{I}$ & $3 / \mathrm{II}$ & $9 / \mathrm{II}$ & $22 / \mathrm{II}$ & $8 / \mathrm{III}$ \\
\hline 1 & 0.0 & 0.0 & 0.1 & 0.1 & 0.2 & 0.2 & 0.4 \\
2 & 0.0 & 0.1 & 0.2 & 0.3 & 0.3 & 0.4 & 0.4 \\
3 & 0.0 & 0.1 & 0.4 & 0.5 & 0.9 & 0.9 & 1.3 \\
4 & 0.1 & 0.5 & 0.6 & 0.8 & 1.2 & 0.9 & 1.5 \\
5 & 0.0 & 0.1 & 0.5 & 0.7 & 1.3 & 1.6 & 2.0 \\
6 & 0.0 & 0.3 & 0.7 & 0.9 & 1.1 & 1.5 & 2.2 \\
7 & 0.1 & 0.8 & 2.2. & 3.5 & 6.8 & 10.1 & 15.3 \\
8 & 0.1 & 1.0 & 3.4 & 5.8 & 12.5 & 25.9 & 36.1 \\
\hline
\end{tabular}




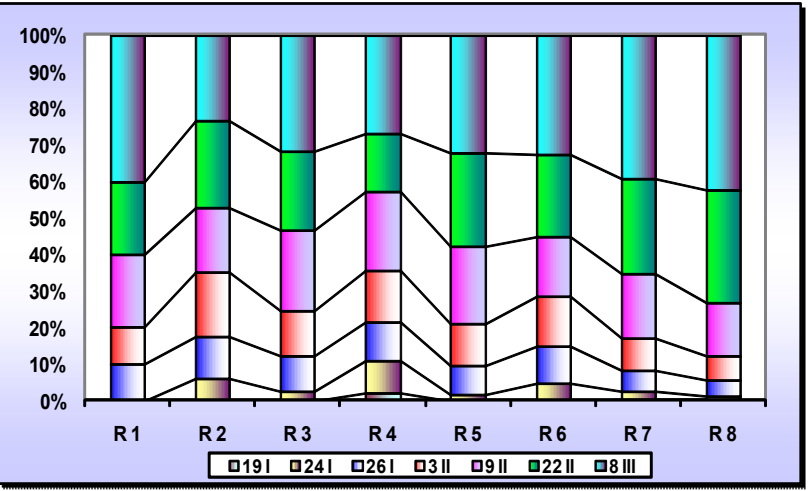

Fig. 3.Percentage of growth of the branches

All the graphs represent the final average length of the branches, in $\mathrm{cm}$, resulting from the buds of rows 5, 6, 7 and 8 .

The witness/control has a similar pattern to growth characteristics of series of batch 1 (Fig. 4), and resulted in longest branches.

\section{Batch 1-Disbudding of the buds of rows 7 and 8}

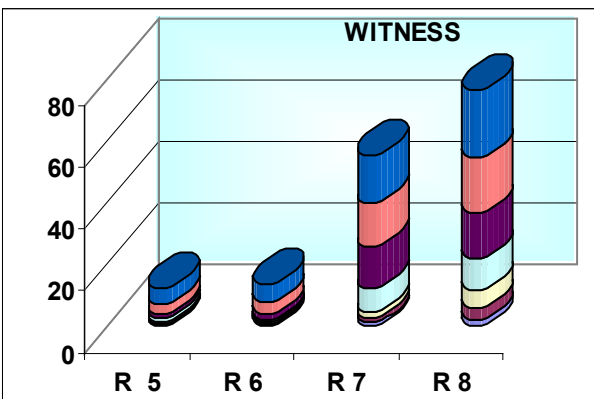

$\square 8$ III

$\square 22$ II

$\square 9$ II

$\square 3$ II

$\square 26$ I

$\square 24$ I

$\square 19 \mathrm{I}$

Fig. 4. The final average length of the branches, in $\mathrm{cm}$, for witness

When the buds of rows 7 and 8 are removed, the buds of rows 5 and 6 displayed a higher growth than that of the witness/control (Fig. 5), meaning the end buds of the vine shoot inhibit the growth of the subjacent buds.

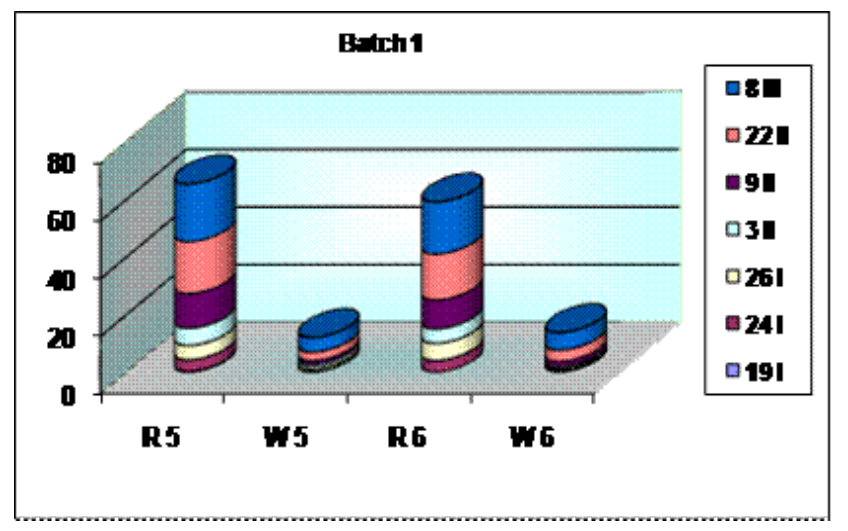

Fig. 5. The final average length of the branches, in $\mathrm{cm}$, for batch 1
Batch 2-Disbudding of the buds of rows 5 and 6

The removal of the buds of rows 5 and 6 was ineffective on growth of the buds of rows 7 and 8 (Fig. 6), which emphasizes that the subjacent buds were not influential on the overlying buds. Inhibition would be thus a polarized phenomenon basipete.

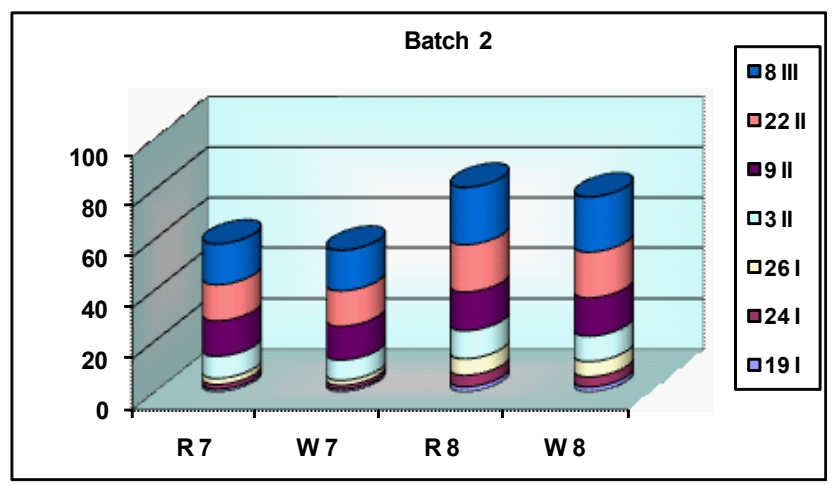

Fig. 6. The final average length of the branches, in $\mathrm{cm}$, for batch 2

Batch 3-Disbudding of the buds of row 8

With the removal of end buds, the growth of $5^{\text {th }}$ and $7^{\text {th }}$ buds was not altered and results in similar length, while the growth of bud 6 was significantly induced in comparison to the witness/control (Fig. 7). It could be supposed that the overlying bud exerts when subjacent bud was inhibited, but only on the same orthostic.

\section{Batch 4-Disbudding of the buds of row 7}

After disbudding of row 7 , the growth of bud 5 showed a significant increase while that of the buds row 8 and 6

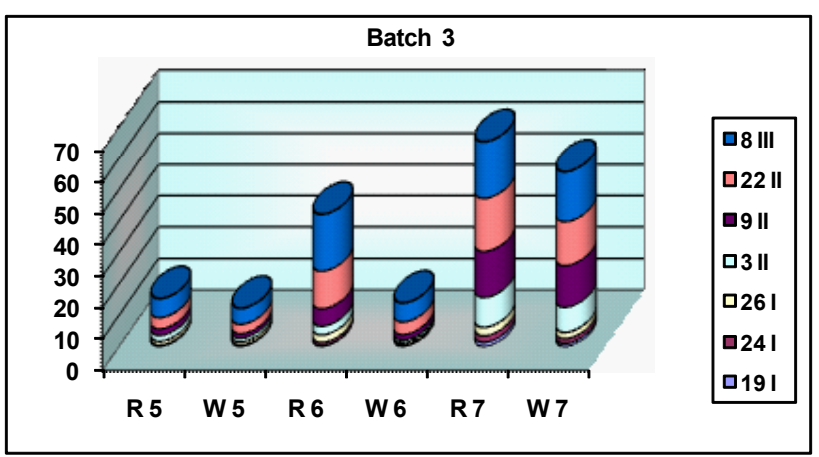

Fig. 7. The final average length of the branches, in $\mathrm{cm}$, for batch 3

was similar to control (Fig. 8), which meaning that growth correlations were effective only on the same orthostic and inhibition was sectorial, similar to results of batch 3 .

\section{Batch 5-Disbudding of the buds of row 6}

The removal of row 6 did not alter the growth of the buds in rows 7 and 8 (Fig. 9). There was no inhibition by the subjacent buds. The growth of the bud of row 5 was not affected since it was not on the same orthostic. 
90

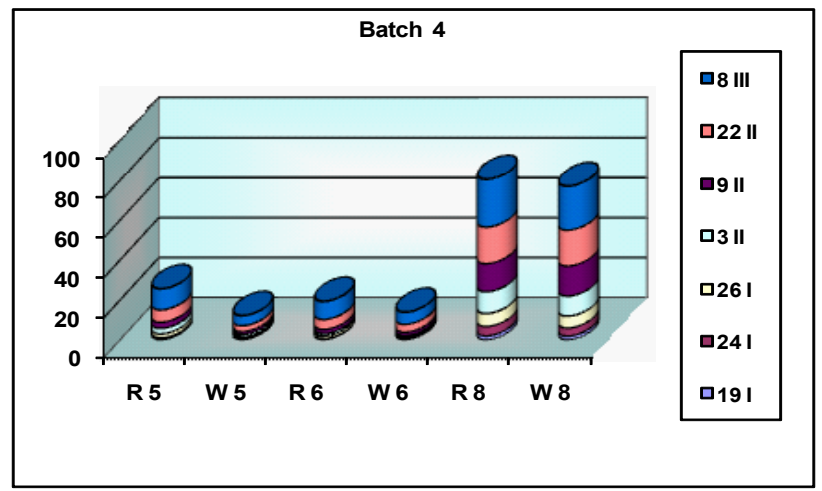

Fig. 8. The final average length of the branches, in $\mathrm{cm}$, for batch 4

Batch 6-Disbudding of the buds of row 5

The removal of the bud of row 5 did not influence the growth of other buds (Fig. 10) confirming that subjacent buds had no significant inhibition and thus inhibition was polarized basipet.

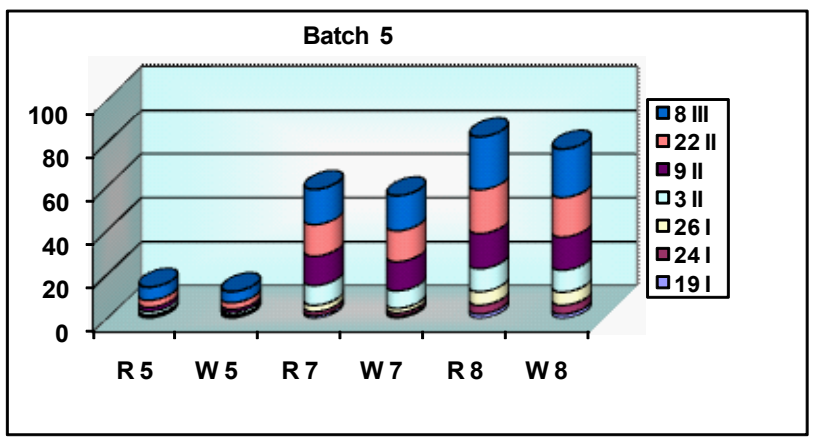

Fig. 9. The final average length of the branches, in $\mathrm{cm}$, for batch 5

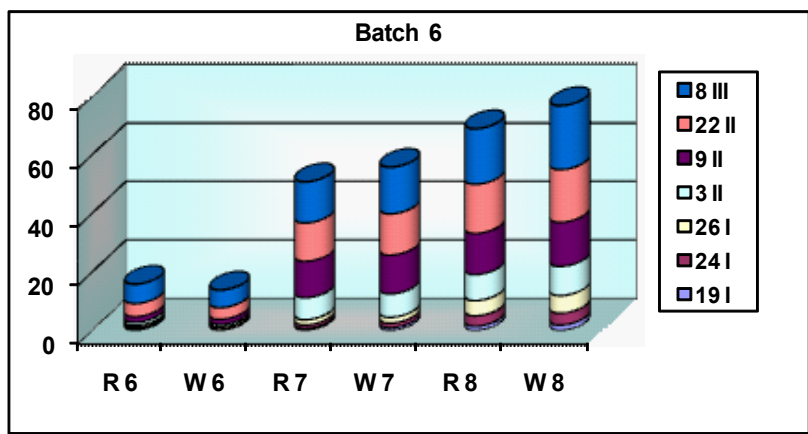

Fig. 10. The final average length of the branches, in $\mathrm{cm}$, for batch 6

\section{Batch 7-Disbudding of the buds of row 8 and application of an auxine}

When bud 8 is disbudded, the growth of bud 6 was stimulated since there was no inhibition (Fig. 11). However, the application of ANA, with $10^{-3} \mathrm{~g} / \mathrm{L}$, to wound the effects of disbudding, the growth was not that significant. This observation mentioned that the auxin was a mediator of the inhibition and migrated within the cutting in a polarized way through basipet and sectorial. However, inhibition was partial as the presence of bud 8 was influen-

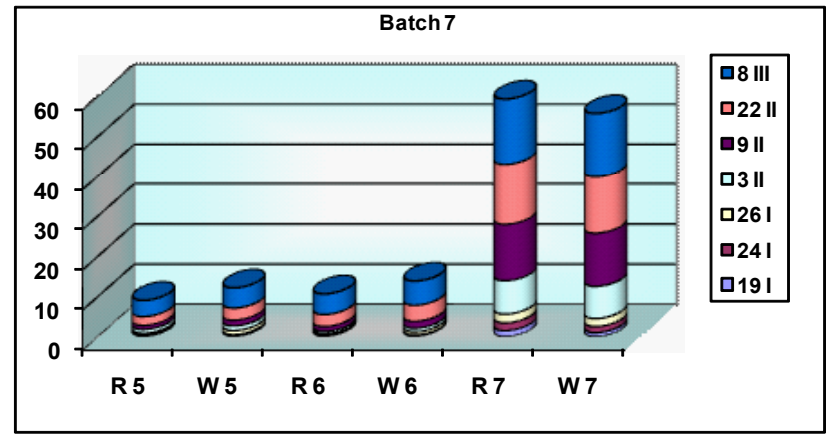

Fig. 11. The final average length of the branches, in $\mathrm{cm}$, for batch 7

tial on inhibition. This could be explained by the fact that the quantity of ANA absorbed by the vine shoot was lower than that applied, according to the size of the wound and of the absorption capacity.

According to Tardea (1995) a given start-up starts to show a correlative inhibition on the subjacent buds or the growth when it reaches a 1-2 cm length. The application of auxin causes a reduction in branch growth resulting from the activity of subjacent bud and a strong bud inhibition for the lower rows.

\section{Conclusions}

The correlations of growth define two great principles: the acrotony of budding and the acrotonie of growth. Ultimately, the mode of ramification basically acrotone of the vine appears to be due as well to properties fixed as at action "current". The fixed properties are responsible for the differences in growth at the appearing beginning of budding are discredit of the buds of row 1 and 2. It is those which have the least complex structure.

Passed this first phase of growth, it still persist a share of property by the fact of this variation in relative value of increase in growths. But especially are added "current" inhibitions which have like properties a polarized inhibition basipete and sectorial. This dernier physiological phenomenon can be explained by the anatomical support of the branch. The development is sectorial because the buds are dependent the ones on the others but only on the same orthostique.

Indeed, the buds communicate between-them by a unilateral criblo-vascular way, i.e. without exchange from one orthostique to another. The auxine is one of the mediators contributing to the sectorial phenomenon. To become of buds will depend on the level of development which it will have acquired for the period when appear the fixed properties.

The correlations of growth thus have a real impact on the farming techniques. Indeed, one of the applications to the vineyard is the size: bowing makes it possible to develop the median buds by more feeding them in sap; the 
size runs (in 2 eyes) allows more balanced growth of the buds left the size of winter.

\section{Acknowledgements}

This study was financed by the Executive Unit for Higher Education Financing and Scientifically Research, under project PCE-IDEI, with code CNCSIS 1141.

\section{References}

Adrian, M., J. C. Fournioux and R. Bessis, (1996). In vitro morphogenesis of grapevine (Vitis vinifera L.) originated from anticipated or latent buds. Vitis 35:107-11.

Bell, A. (1991). Plant form-an illustrated guide to flowering plant morphology. Oxford University Press, Oxford, UK.

Bessis, R. (1965). Recherches sur la fertilité et le corrélations de croissance entre bourgeons chez la vigne (Vitis vinifera L.). Université de Bourgogne Dijon, Thése d'état, Abstr.

Bessis, R. (1986). Grapevine physiology: the contributuin of culture in vitro. Cellular and Molecular Life Sciences 42 (8): 927-933.

Bessis, R. and J. C. Fournioux, (1992). Zone d'abscission et coloure de la vigne. Vitis 31:9-21.

Bessis, R., N. Charpentier, C. Hilt, J. C. Fournioux (2000). Grapevine fruit set: phsiology of the abscission zone. Australian Journal of Grape and Wine Research 6: 125130.

Champagnol, F. (1984). Elements de physiologie vegetale et de viticulture generale. Ed. Tech and Doc., Paris.

Fournioux, J. C. (1998). Foliar effects in the controlled development of anticipated buds of Vitisvinifera L. Canadian Journal of Botany 76:1385-1403.

Fournioux, J. C. (2001). Demonstration of a correlation between young leaves, apex and young brunches specific to young grapevine cutting in the first period of its development. Journal International des Sciences de la Vigne et du Vin 35:117-127.
91

Galet, P. (1993). Précis de viticulture, $6^{\mathrm{eme}}$ édition. Ed. Tech and Doc., Montpellier.

Goffinet, M. (1991). Buds and shoots of grapevines. Grape Research News 2:1-3.

Huglin, P. (1998). Biologie et écologie de la vigne. $2^{\text {eme édition. }}$ Ed. Tech and Doc., Paris.

Lauri, P. E. (2007). Differentiation and growth traits associated with acrotony in the apple tree (Malus domestica, Rosaceae). American Journal of Botany 94:1273-1281.

Lebon, E., A. Pellegrino, F. Tardieu, J Lecoeur. (2004). Shoot development in grapevine (Vitis vinifera) is affected by modular branching pattern of the stem and intra- and intershoot trophic competition. Annals of Botany 93:263-274.

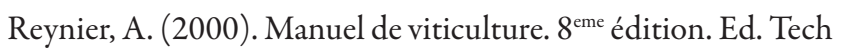
and Doc., Paris.

Tardea, C., Dejeu, L. (1995). Viticultura. Ed. Didactica si Pedagogica, Bucuresti.

Vasconcelos, M. C., M. Greven, C. S. Winefield, M. C. T. Trought and V. Raw (2009). The flowering of Vitis vinifera: a review. American Journal of Enology and Viticulture 60(4):411-434.

Vincent, G. (1995). Étude expérimantale de la ramification acrotone d'un Pelargonium ligneux (geranium roast). Canadian Journal of Botany 73:1557-1566.

Williams, L. E. (2000). Bud development and frutfulness of grapevines. In: Raisin production manual (L. P. Christensen). pp 24-29. University of California Division of Agriculture and Natural Resources, Oakland. 